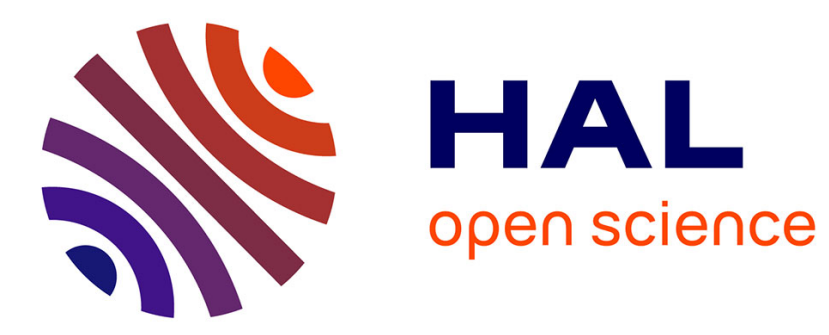

\title{
Indexation of Electron diffraction patterns at grain boundaries
}

\author{
Qiwei Shi, Ying Zhou, Hongru Zhong, Dominique Loisnard, Chengyi Dan, \\ Fengguo Zhang, Zhe Chen, Haowei Wang, Stéphane Roux
}

\section{To cite this version:}

Qiwei Shi, Ying Zhou, Hongru Zhong, Dominique Loisnard, Chengyi Dan, et al.. Indexation of Electron diffraction patterns at grain boundaries. Materials Characterization, In press. hal-03410865

\section{HAL Id: hal-03410865 https://hal.science/hal-03410865}

Submitted on 1 Nov 2021

HAL is a multi-disciplinary open access archive for the deposit and dissemination of scientific research documents, whether they are published or not. The documents may come from teaching and research institutions in France or abroad, or from public or private research centers.
L'archive ouverte pluridisciplinaire HAL, est destinée au dépôt et à la diffusion de documents scientifiques de niveau recherche, publiés ou non, émanant des établissements d'enseignement et de recherche français ou étrangers, des laboratoires publics ou privés. 


\title{
Indexation of Electron diffraction patterns at grain boundaries
}

\author{
Qiwei Shi ${ }^{1,2}$, Ying Zhou ${ }^{1}$, Hongru Zhong ${ }^{1}$, Dominique Loisnard ${ }^{3}$, Chengyi Dan ${ }^{1}$, \\ Fengguo Zhang ${ }^{1 *}$, Zhe Chen ${ }^{1 *}$, Haowei Wang ${ }^{1}$, Stéphane Roux ${ }^{4}$ \\ 1: School of Materials Science and Engineering, \\ Shanghai Jiao Tong University, Shanghai, 200240, China \\ 2: SJTU-Paris Elite Institute of Technology, \\ Shanghai Jiao Tong University, Shanghai, 200240, China \\ 3: EdF R\&D, Site des Renardières, avenue des Renardières, \\ Ecuelles, F-77818 Moret-sur-Loing, France \\ 4: Université Paris-Saclay, ENS Paris-Saclay, CNRS, \\ LMT - Laboratoire de Mécanique et Technologie, F-91190, Gif-sur-Yvette, France \\ Received: date / Accepted: date
}

\begin{abstract}
Grain boundaries play a vital role in materials science, and they are extensively studied through experimental and numerical methods. Electron backscatter diffraction (EBSD) is commonly used to characterize crystalline materials, yet its performance is often compromised at grain boundaries. The key difficulty is to index the overlapped electron backscatter patterns, where Kikuchi bands from multiple crystal orientations coexist. Houghindexation often fails in treating overlapped EBSP, while the recently proposed dictionary indexation and sphere indexation give only one triplet of Euler angles from overlapped patterns. For all these methods, the orientation indexing precision drops for overlapped EBSP. Here we propose an integrated Digital Image Correlation (DIC) procedure to determine simultaneously and precisely multiple crystal orientations by registering the overlapped EBSP and the master pattern. The contribution ratio of each crystal orientation to the overlapped pattern is also available. Through an experimental EBSD dataset around a triple point, multiple benefits of the rich results from the method are demonstrated, such as refining grain boundaries, revealing EBSD scan errors and quantifying spatial resolution of EBSD. At the grain boundaries, the crystal orientation measurement uncertainty is $0.03^{\circ}$ for both indexed crystal orientations, the same order for grain interiors, while their contribution ratios of uncertainty around 0.02 are obtained.
\end{abstract}

Keyword: Integrated digital image correlation, Crystal orientation, Overlapped pattern, Grain boundary, High-angular-resolution EBSD, Interaction volume. 


\section{Introduction}

Grain boundaries have long drawn attention of scientists and engineers of the fields of materials, mechanics, chemistry etc. For example, very often the cracks are initiated from grain boundaries [1, severe corrosion attack tend to take place there [2, some activated slip systems may transfer across grain boundaries, otherwise incompatibilities lead to dislocation accumulation [3. Characterizing precisely the microstructure at grain boundaries is the preliminary step for all these studies, and indexing the crystal orientations is a key aspect.

In recent decades backscattering electron diffraction (EBSD technique) revealed to be a method of choice for the measurement of crystallographic orientation fields in scanning electron microscopes. Multiple algorithms have been adopted to treat electron backscatter patterns(EBSP) and extract crystal orientations, such as the Hough transformation 4, dictionary indexing [5, 6, 7, spherical indexing [8], convolutional neural network [9, 10]. The Hough-transformation method has been used overwhelmingly in commercial common EBSD acquisitions. Though demanding in computing capacity, dictionary indexing has proven very robust in presence of high noise level [11, and is able to distinguish phases of the same Bravais lattice [12].

Full pattern matching method has also been applied to higher-resolution EBSPs to calibrate EBSD acquisition and refine crystal orientations. The basic idea is to tune the projection parameters (and in particular the three coordinates of the Projection Center, PC) in order to maximize the similarity between experimental and simulated EBSPs. Two software packages are available for dynamical EBSP simulations, namely EMsoft [13] and ESPRIT DynamicS from Bruker Nano GmbH. To achieve the optimization of the projection parameters, many algorithms have been suggested, such as a Nelder-Mead algorithm [14, 15, evolution algorithm [16], SNOBFIT algorithm [15, Integrated DIC method [17] as well as non-disclosed ones used in commercial software [18]. These methods, with an angular resolution in the order of $0.03-0.05^{\circ}$ and a PC precision in the order of $0.1 \%$ of EBSP width, help to reveal the fine structures of materials [19, 17].

Inevitably, electron beams sometimes hit grain boundaries and produce overlapped EBSPs, which contain diffraction information from multiple crystal orientations. The smaller the grain size, (e.g., for most high-performance engineering metals), the higher the probability to get overlapped EBSPs. Though rich in information, overlapped EBSPs pose a difficult question for all the previously mentioned indexing methods, especially for the commonly used Hough-transformation algorithm [20].

The effect of overlapped EBSP at grain boundaries has been addressed in previous studies. Cross-correlation has been used to seperate the contributions of different crystal orientations, leading to more precise strain and rotation measurements [21. However, the eventual shift of Kikuchi bands between the reference pattern and the overlapped pattern poses a severe problem, thus the method has neglected the aforementioned shift, which may not hold for general EBSD cases. The orientation indexing precision at grain boundaries is evaluated in Ref. [20, in order to attribute higher KAM values near grain boundaries either to the real misorientation or to the increased indexation error from overlapped EBSPs. Tong et al. 22] have systematically studied the effect of overlapped EBSPs on High-resolution EBSD (HR-EBSD) measurements. They showed that the HR-EBSD accuracy is only affected when the indexed point is very close to grain boundaries, say about $20 \mathrm{~nm}$. However, it is difficult to separate the overlapped EBSPs by the cross-correlation method when strain/rotation is present, and the natural 
choice is to filter out the Kikuchi bands which contribute less. In this way the algorithm manages to get one crystal orientation and eventually the elastic strain levels. As the dominating Kikuchi bands lead to a higher match, the dictionary indexing method gets the corresponding crystal orientation from an overlapped EBSP without specific processing [23. Therefore, for both methods a large part (as high as 50\%) of the EBSP information could be lost. The discarded secondary Kikuchi lines could deteriorate the precision of indexation, while they would enrich the EBSD analysis if properly treated. Recently Lenthe et al. proposed a strategy that relies on overlapped master patterns to index crystal orientations from overlapped EBSPs, however their method has only been applied to orientation-related phases [24. Therefore, there exists no algorithm that is applicable to overlapped EBSPs for random crystal orientations.

Recently, an Integrated Digital Image Correlation (IDIC) algorithm to calibrate EBSD tests was proposed [17. The method gives Euler angles and projection center coordinates simultaneously with a high precision. A proper cost function that incorporates the noise level of experimental EBSPs is proposed to enhance the calibration precision. Newton's method has been implemented in the optimization, resulting in rapid convergences. The algorithm is also highly tunable and the number of projection parameters can be modified freely to adapt to different scenarios.

In the present paper the IDIC EBSD calibration algorithm [17] is extended to process overlapped EBSPs, including refining multiple Euler angle sets, one projection center, and estimate the contribution percentage of each Euler angle set. Section 2 introduces the chosen notations, including the projection parameters for multiple crystals. Then, the IDIC data processing method for overlapped EBSPs indexation is detailed. Section 3 shows the algorithm performance on high-resolution EBSPs of a polycrystal Al alloy. The implementation of overlapped EBSP indexation enhances significantly crystal orientation precision, and minute EBSD scan errors can be visualized thanks to their influence on indexation. Section 4 studies a strained polycrystal $\mathrm{Al}-\mathrm{Mg}$ alloy, and tests the performance of the algorithm on a dataset with orientation gradients. The indexation of multiple crystal orientations from overlapped patterns turns out to be highly beneficial from many points of view.

\section{$2 \quad$ Integrated DIC for indexing overlapped EBSP}

The Integrated Digital Image Correlation (IDIC) has been proposed to measure directly the physical quantities (parameters) of interest from image registrations [25, 26, 27. The measured displacement fields between reference and target images are expressed in a "kinematic basis" that is tailored a priori to the studied physical phenomena, and potentially the artefacts of image formation and acquisition. Besides, IDIC provides a residual field, which is calculated at each pixel and constitutes by nature a good criterion to characterize the registration error, as will be demonstrated in Section 3. For example, IDIC framework have been adopted in high-resolution EBSD to estimate elastic strains and quantify the optical distortions in EBSP formation [28, 29, 30, 31.

A new IDIC-based algorithm has been developed to index overlapped EBSPs which is detailed in this section. The projection geometry of EBSD patterns is recalled in figure 1 together with the reference coordinate system used in the paper. IDIC can be adapted to index overlapped EBSPs since it is based on an interpretative framework adjusted to the expected kinematics. 


\subsection{Algorithm}

In the IDIC EBSD calibration algorithm, 6 projection parameters are assumed to be necessary for producing a specific EBSP, including the coordinates of the PC (shown with a red star in Figure 1) and the crystal orientation expressed as Euler angles. All other factors are neglected, such as elastic/plastic strains, in-homogeneous energy distribution of the diffracted electrons [32, the Scanning Electron Microscope (SEM) distortion and EBSP background. In spite of these simplifying assumptions, it was shown that good calibrations results could be obtained, such as fast convergence, precise calibration parameters and high tolerance to a rough surface, albeit a PC calibration error about $0.1 \%$ of EBSP width could exist for strained samples [17.

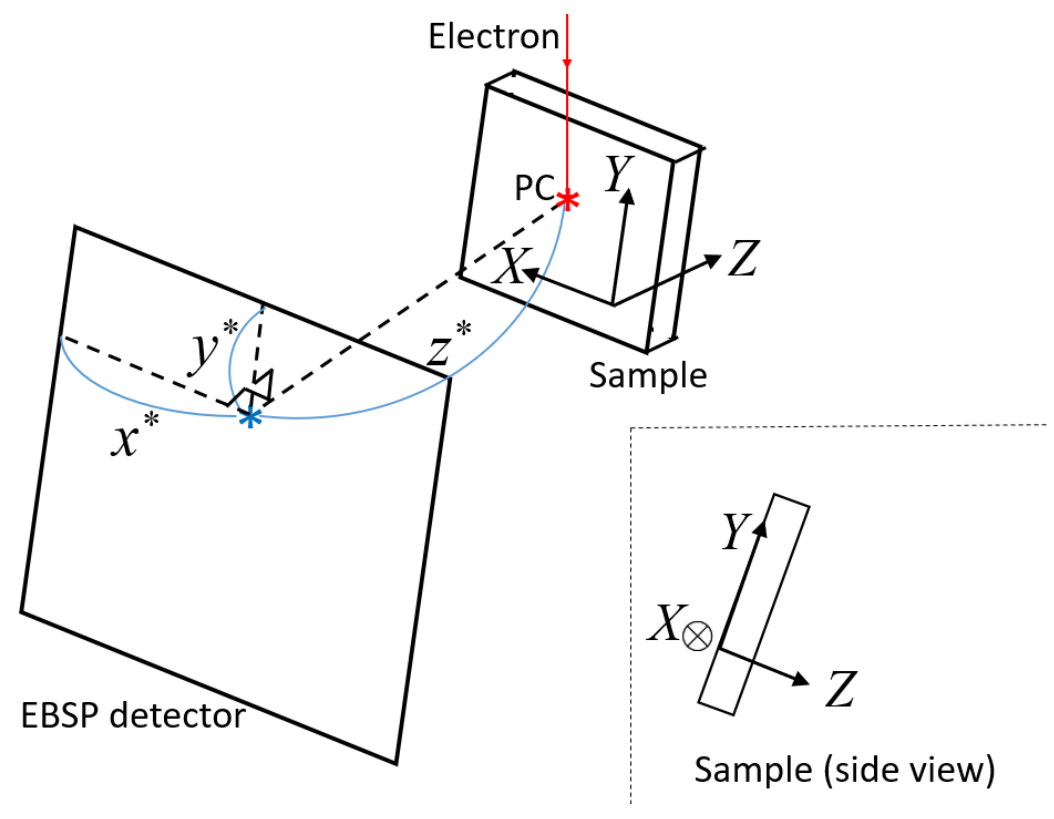

Figure 1: Coordinate systems $(x, y, z)$ for the EBSP detector and $(X, Y, Z)$ for the sample.

In the present algorithm for overlapped EBSPs, one set of 3 Euler angles per grain is necessary. In this section, the case of two grains is considered first and Euler angles are denoted as $\left(\varphi_{11}, \phi_{1}, \varphi_{12}\right),\left(\varphi_{21}, \phi_{2}, \varphi_{22}\right)$. As the interaction volume size is negligible compared to EBSP detector pixel size (generally larger than $20 \mu \mathrm{m}$ ), the projection center $\left(x^{*}, y^{*}, z^{*}\right)$ can still be assimilated to a single point for the interaction volume at grain boundary. Besides, a parameter $\lambda$ indicating the contributing percentage of the two crystal orientations is introduced. As a result, the column vector $\boldsymbol{P}$ gathering all parameters for overlapped EBSP indexation consists of 10 parameters $\left(\varphi_{11}, \phi_{1}, \varphi_{12}, \varphi_{21}, \phi_{2}, \varphi_{22}, x^{*}, y^{*}, z^{*}, \lambda\right)^{T}$.

The mathematical formula relating EBSP to projection parameters remain identical to that described in ref. [17. The rotation matrix based on the two Euler angle sets reads in Bunge notations:

$$
\boldsymbol{Q}_{1}=\left[\begin{array}{ccc}
\cos \varphi_{11} \cos \varphi_{12}-\sin \varphi_{11} \sin \varphi_{12} \cos \phi_{1} & \sin \varphi_{11} \cos \varphi_{12}+\cos \varphi_{11} \sin \varphi_{12} \cos \phi_{1} & \sin \varphi_{12} \sin \phi_{1} \\
-\cos \varphi_{11} \sin \varphi_{12}-\sin \varphi_{11} \cos \varphi_{12} \cos \phi_{1} & -\sin \varphi_{11} \sin \varphi_{12}+\cos \varphi_{11} \cos \varphi_{12} \cos \phi_{1} & \cos \varphi_{12} \sin \phi_{1} \\
\sin \varphi_{11} \sin \phi_{1} & -\cos \varphi_{11} \sin \phi_{1} & \cos \phi_{1}
\end{array}\right]
$$




$$
\boldsymbol{Q}_{2}=\left[\begin{array}{ccc}
\cos \varphi_{21} \cos \varphi_{22}-\sin \varphi_{21} \sin \varphi_{22} \cos \phi_{2} & \sin \varphi_{21} \cos \varphi_{22}+\cos \varphi_{21} \sin \varphi_{22} \cos \phi_{2} & \sin \varphi_{22} \sin \phi_{2} \\
-\cos \varphi_{21} \sin \varphi_{22}-\sin \varphi_{21} \cos \varphi_{22} \cos \phi_{2} & -\sin \varphi_{21} \sin \varphi_{22}+\cos \varphi_{21} \cos \varphi_{22} \cos \phi_{2} & \cos \varphi_{22} \sin \phi_{2} \\
\sin \varphi_{21} \sin \phi_{2} & -\cos \varphi_{21} \sin \phi_{2} & \cos \phi_{2}
\end{array}\right]
$$

As in the previously proposed IDIC EBSD calibration algorithm, the EBSP screen is chosen as the reference defining the $(x, y)$ plane, or $z=0$. The unit vector $\boldsymbol{w}$ linking the projection center to each pixel $\boldsymbol{x}=(x, y, z=0)$ of the screen can be expressed as

$$
\boldsymbol{w}=\frac{\left(\boldsymbol{x}-\boldsymbol{x}^{*}\right)}{\left|\boldsymbol{x}-\boldsymbol{x}^{*}\right|}
$$

In order to rotate this vector from the screen frame of reference $\boldsymbol{w}$ to that of the spherical master pattern, $\boldsymbol{v}$, one should multiply it by the rotation operators $\boldsymbol{Q}_{1}$ and $\boldsymbol{Q}_{2}$.

$$
\boldsymbol{v}_{1}=\boldsymbol{Q}_{1} \boldsymbol{w}, \boldsymbol{v}_{2}=\boldsymbol{Q}_{2} \boldsymbol{w}
$$

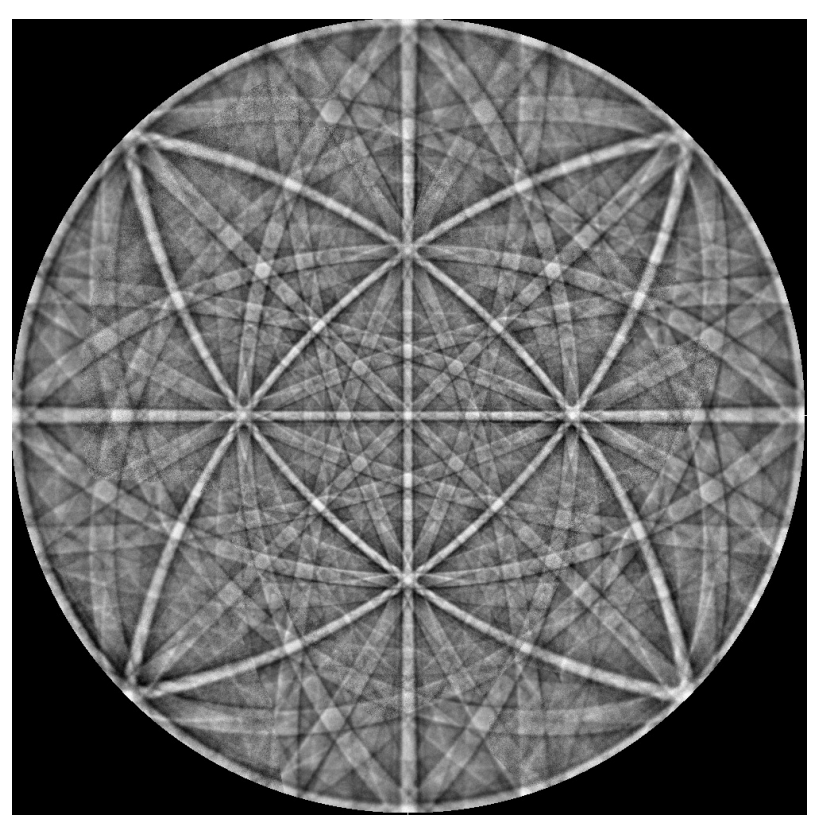

Figure 2: Master pattern generated by projecting 50 EBSPs back to $\boldsymbol{u}$ domain.

EMsoft generates a master pattern by dynamical simulation, which can be projected onto 2D detectors to generate EBSPs of any crystal orientation. The master pattern is originally defined on the surface of a sphere centered on the interaction volume. Several projection methods have been adopted in the EBSD simulation community, such as spherical projection and Lambert projection, in order to represent the master pattern efficiently as a 2D matrix. The spherical projection of the master pattern was adopted in IDIC-EBSD, due to its simpler projection formula.

Experimental EBSPs can also be projected back to form master patterns [33, 34]. Figure 2 shows the master pattern created by projecting back 50 experimental EBSPs, which are hand-picked to cover each grain of an EBSD acquisition using the same experimental setup reported in Refs. [17, 35. In this way, the master pattern has the same gray level distribution as experimental EBSPs. This master pattern is used throughout the present paper to generate 
simulated EBSPs for any crystal orientation, including the two different Kikuchi band systems of an overlapped EBSP. Note that the proposed method is intrinsically different from that of Ref. 24, which calculates overlapped master patterns for certain orientation-related phases before indexation. As a result, the present algorithm is less demanding in computation load and provides a very good flexibility. It is vital to use the master pattern generated from experimental EBSPs, rather than a dynamically simulated master pattern. If not, $\lambda$ values tend to concentrate around 0.3-0.7, even if the indexed point is far from grain boundaries. This shows that with more degrees of freedom to determine, we need to make the gray levels as similar as possible.

The spherically projected points $\left(\boldsymbol{u}_{1}, \boldsymbol{u}_{2}\right)$ on the master pattern are expressed as:

$$
\boldsymbol{u}_{1}=\frac{\boldsymbol{n}-\boldsymbol{v}_{1}}{\left(1-\boldsymbol{v}_{1} . \boldsymbol{n}\right)}-\boldsymbol{n}, \boldsymbol{u}_{2}=\frac{\boldsymbol{n}-\boldsymbol{v}_{2}}{\left(1-\boldsymbol{v}_{2} . \boldsymbol{n}\right)}-\boldsymbol{n}
$$

where $\boldsymbol{n}$ denotes the unit vector pointing at the north pole: $\boldsymbol{n}=(0,0,1)^{\top}$. Thus the projected position $\boldsymbol{u}_{1}$ and $\boldsymbol{u}_{2}$ can be explicitly expressed as a function of $\left(\varphi_{11}, \phi_{1}, \varphi_{12}, x^{*}, y^{*}, z^{*}\right)$ or $\left(\varphi_{21}, \phi_{2}, \varphi_{22}, x^{*}, y^{*}, z^{*}\right)$. The expression is quite long but presents no difficulty (can be easily calculated by formal calculus software such as Matlab or Mathematica), and is thus not presented here. An illustration of the relationship between the 4 adopted spaces $\boldsymbol{x}, \boldsymbol{w}, \boldsymbol{v}, \boldsymbol{u}$ can be found in Ref. [17.

Conventional DIC consists in correlating two gray level images, one of the reference state $f(\boldsymbol{x})$ and the other one of the deformed state $g(\boldsymbol{x})$. In the herein proposed algorithm, experimental overlapped EBSP is taken as $f(\boldsymbol{x})$ and the EBSP master pattern $g(\boldsymbol{u})$. Therefore the simulated EBSPs of the two crystal orientations correspond respectively to $g_{\boldsymbol{u}_{1}}(\boldsymbol{x})=g\left(\boldsymbol{u}_{1}(\boldsymbol{x} ; \boldsymbol{P})\right)$ and $g_{\boldsymbol{u}_{2}}(\boldsymbol{x})=g\left(\boldsymbol{u}_{2}(\boldsymbol{x} ; \boldsymbol{P})\right) . g_{\boldsymbol{u}_{1}}(\boldsymbol{x})$ and $g_{\boldsymbol{u}_{2}}(\boldsymbol{x})$ overlap to form a superposed EBSP, and a parameter $\lambda$ can be introduced to characterize the contribution of the two crystal orientations. The overlap of Kikuchi bands is simulated as a mere linear combination, as proposed in Refs. [21, 20, 24].

$$
g_{\boldsymbol{u}}(\boldsymbol{x})=\lambda g_{\boldsymbol{u}_{1}}(\boldsymbol{x})+(1-\lambda) g_{\boldsymbol{u}_{2}}(\boldsymbol{x})
$$

The IDIC algorithm involves matching at best $f(\boldsymbol{x})$ and $g_{\boldsymbol{u}}(\boldsymbol{x})$, through the minimization of a quadratic norm of the residual $r$ summed over the entire ROI. The cost function to minimize writes

$$
\Theta=\sum_{R O I} \omega(\boldsymbol{x})^{2}\left[f(\boldsymbol{x})-g_{\boldsymbol{u}}(\boldsymbol{x})\right]^{2}
$$

where $\omega(\boldsymbol{x})$ is the weight associated to each pixel $\boldsymbol{x}, g_{\boldsymbol{u}}(\boldsymbol{x})$ is the current estimate of simulated overlapped EBSP during iterative algorithms. The weights $\omega$ are introduced here as they may be used to make this functional optimal with respect to the handling of noise. This point has been made explicit in Ref. [17, and those $\omega(\boldsymbol{x})$ fields are reused in the present study. The minimization of the cost function leads to successive corrections of the transformations $\boldsymbol{u}_{1}(\boldsymbol{x})$ and $\boldsymbol{u}_{2}(\boldsymbol{x})$ until convergence [36. Starting from an approximate solution, the transformations $\boldsymbol{u}_{1,2}(\boldsymbol{x})$ is progressively corrected with linear combinations of sensitivity fields constituting the kinematic basis. For the proposed algorithm, IDIC is the chosen tool to register diffraction images.

\subsection{Solution}

The minimization algorithm follows the standard IDIC steps without particular modification [36. The cost function (7) is iteratively minimized with a Gauss-Newton algorithm. 
A slight modification of parameter $\left(\varphi_{11}, \phi_{1}, \varphi_{12}\right)$, or $\delta P_{i=1,2,3}$, induces a modification of the corrected deformed image as

$$
\delta g_{\boldsymbol{u}}(\boldsymbol{x})=\lambda \nabla g(\boldsymbol{u}) \cdot \frac{\partial \boldsymbol{u}_{1}(\boldsymbol{x} ; \boldsymbol{P})}{\partial P_{i=1,2,3}} \delta P_{i=1,2,3}
$$

Similarly, for infinitesimal modification of $\left(\varphi_{21}, \phi_{2}, \varphi_{22}\right)$, or $\delta P_{i=4,5,6}$, the corrected deformed image changes

$$
\delta g_{\boldsymbol{u}}(\boldsymbol{x})=(1-\lambda) \nabla g(\boldsymbol{u}) \cdot \frac{\partial \boldsymbol{u}_{2}(\boldsymbol{x} ; \boldsymbol{P})}{\partial P_{i=4,5,6}} \delta P_{i=4,5,6}
$$

The infinitesimal modification of $\left(x^{*}, y^{*}, z^{*}\right)$, or $\delta P_{i=7,8,9}$, leads to

$$
\delta g_{\boldsymbol{u}}(\boldsymbol{x})=\left[\lambda \nabla g(\boldsymbol{u}) \cdot \frac{\partial \boldsymbol{u}_{1}(\boldsymbol{x} ; \boldsymbol{P})}{\partial P_{i=7,8,9}}+(1-\lambda) \nabla g(\boldsymbol{u}) \cdot \frac{\partial \boldsymbol{u}_{2}(\boldsymbol{x} ; \boldsymbol{P})}{\partial P_{i=7,8,9}}\right] \delta P_{i=7,8,9}
$$

The slight modification of $\lambda$, or $\delta P_{i=10}$, causes

$$
\delta g_{\boldsymbol{u}}(\boldsymbol{x})=\left(g_{\boldsymbol{u}_{1}}(\boldsymbol{x})-g_{\boldsymbol{u}_{2}}(\boldsymbol{x})\right) \delta P_{i=10}
$$

By Newton's method, the column vector $\{\boldsymbol{\delta} \boldsymbol{P}\}$ gathering all corrections to $\boldsymbol{P}$ is obtained

$$
[\boldsymbol{M}]\{\boldsymbol{\delta} \boldsymbol{P}\}=\{\boldsymbol{\gamma}\}
$$

where $[\boldsymbol{M}]$ is the Hessian matrix of size $10 \times 10$ at iteration $n-1$

$$
M_{i j}^{(n-1)} \equiv \frac{\partial^{2} \Theta}{\partial P_{i} \partial P_{j}}
$$

and the second member $\left\{\gamma_{i}\right\}$ includes the residual field

$$
\gamma_{i}^{(n)} \equiv \frac{\partial \Theta}{\partial P_{i}}
$$

The explicit forms of Hessian matrix and the second member can be deduced from Equations 8[11. This procedure presents no special difficulty and is thus not detailed here.

When $\|\{\boldsymbol{\delta} \boldsymbol{P}\}\|<\epsilon, \epsilon$ being chosen equal to $10^{-6}$ for all the calculations of this paper, the minimization stops and $\boldsymbol{P}$ is stored. Otherwise, $\boldsymbol{P}$ is updated

$$
\boldsymbol{P}^{(n)}=\boldsymbol{P}^{(n-1)}+\boldsymbol{\delta} \boldsymbol{P}^{(n)}
$$

Once $\boldsymbol{P}$ is obtained, the simulated EBSP resembles the reference experimental EBSP.

The above explained algorithm is named IDIC-O (Integrated Digital Image Correlation for Overlapped) EBSD indexation method. When $\lambda$ equals 0 or 1, IDIC-O EBSD reduces to the IDIC EBSD algorithm explained in Ref. [17. Note that the 10 parameters listed in IDIC-O EBSD can be tuned freely. For example, the high-frequency part of $3 \mathrm{PC}$ values can be filtered out for smooth-surface samples, or the $\lambda$ value can also be filtered for coarse-grained samples.

The hereby detailed IDIC-O EBSD has targeted overlapped EBSPs of two crystal orientations, while it can be extended to three crystal orientations or even beyond. When a triplet of Euler angles is added in the computation, its contributing factor should be included in $\boldsymbol{P}$, too. As a result, the degrees of freedom is 6 for indexing a single 
orientation, 10 for two orientations, 14 for triple orientations, etc. The construction of Hessian matrix and second member vector follows the same procedure, and presents no special difficulty.

The IDIC-O EBSD algorithm has been implemented in Matlab. The proposed algorithm has been tested on two high-definition EBSD experimental datasets, one at a triple grain joint of an unstrained sample and the other of a strained sample. Both results show the benefits of the algorithm during grain boundary characterization.

\section{Test on an EBSD dataset on a triple grain boundary}

The examined sample is an annealed Aluminium-Magnesium (6\% wt.) alloy, polished and electro-chemically etched. Then a fine EBSD acquisition of step size of $51 \mathrm{~nm}$ was undertaken around a triple-joint grain boundary. A set of $125 \times 169$ EBSPs of resolution $600 \times 800$ pixels and of 8-bit depth were recorded and analysed. Note that the step size was deliberately set small to study the gradual transition from one crystal orientation to another. The Bruker $\mathrm{e}^{-}$Flash $^{H D}$ EBSD detector was mounted on a Tescan MAIA3. The acceleration voltage was $20 \mathrm{kV}$, the probe current $20 \mathrm{nA}$, and the dwell time $0.15 \mathrm{~s}$.

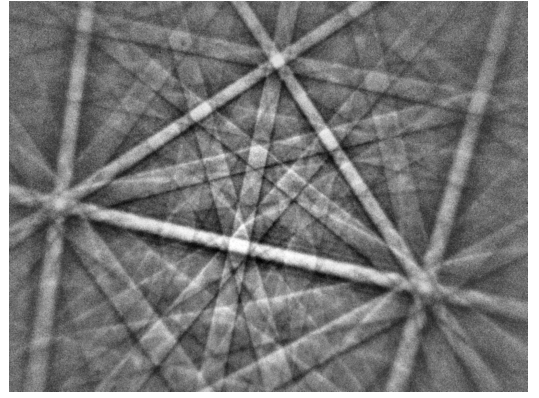

(a)

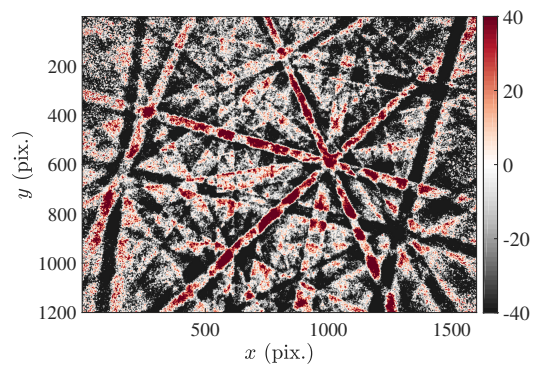

(d)

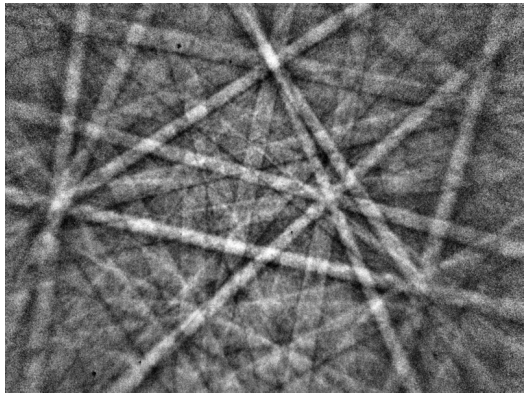

(b)

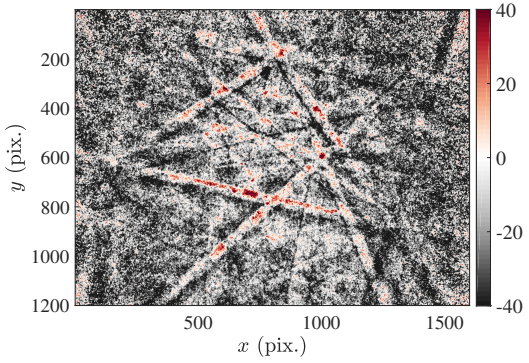

(e)

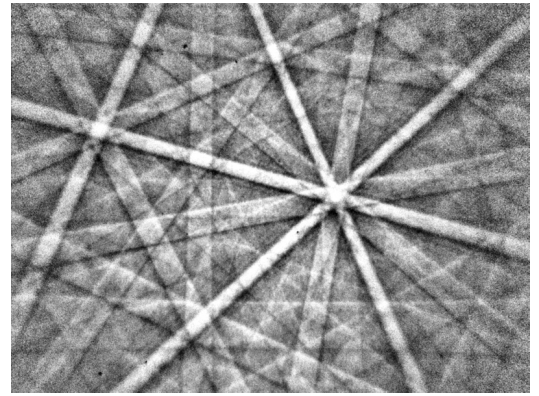

(c)

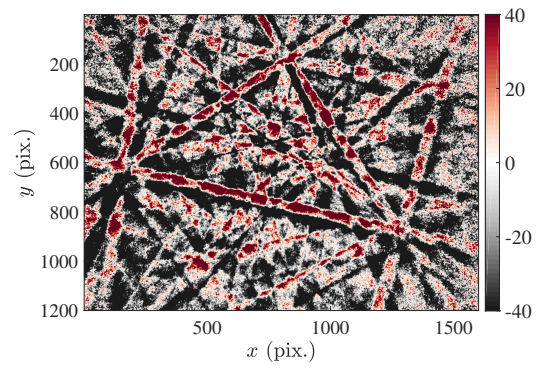

(f)

Figure 3: (a-c) adjacent EBSPs of full resolution $1200 \times 1600$ pixels across a grain boundary. (b) corresponds to an overlapped EBSP containing Kikuchi bands from two crystal orientations. (d-f) show the residual fields of IDIC EBSD indexation of (b), where a white color indicates low residuals and both red and black colors show high residual values (assuming respectively positive and negative values). (d) is initialized with the parameters of (a), (e) initialized with the combined parameters of (a) and (c), and (f) is initialized with the parameters of (c).

Figure 3 shows an example of overlapped EBSP indexation. Figures $3(\mathrm{a})$ 3(c) show three adjacent EBSPs of full resolution $1200 \times 1600$ pixels across a grain boundary. Evidently, the EBSP in the middle is an overlapped pattern 
containing Kikuchi bands of two crystal orientations. The contribution ratio of two Kikuchi bands is roughly halfhalf. Figure $3(\mathrm{~b})$ is indexed using the presented algorithm, and the residual fields are shown in Figure $3(\mathrm{~d})$, $3(\mathrm{f})$ Note that the values in Figure $3(\mathrm{~d})-3(\mathrm{f})$ are to be compared to the largest possible residuals \pm 255 , and values close to zero means a low residual level, thus a good image correlation. Figure $3(\mathrm{~d})$ corresponds to initializing the indexation purely by the parameters retrieved for Figure 3(a) and Figure 3(f) corresponds to residual field when adopting parameters of Figure 3(c). When choosing only one crystal orientation to index an overlapped EBSP, the calculation converges and the chosen Kikuchi bands are properly registered. However, the Kikuchi bands of the other crystal orientation are leftover, and form high (red or black) residuals on Figure 3(d) and Figure 3(f) Figure $3(\mathrm{e})$ shows the residual field when taking both crystal orientations into consideration, and the residuals are much lower, i.e., close to zero. This test case shows clearly the effectiveness in indexing multiple crystal orientations.

The SE image of the indexed area is shown in Figure 4(a). Faint parallel lines are visible on the SE image, and they result from the last HR-EBSD acquisition as reported in Ref. 17]. Figure 4(b)] shows the inverse pole figure given by Bruker and several EBSPs of the fine-step EBSD acquisition around a triple joint. The EBSPs from the three grains and their mutually overlapped EBSPs are shown around the IPF map. An EBSP taken at the triple point, which contains the Kikuchi bands of all three crystal orientations, is also shown in the figure.

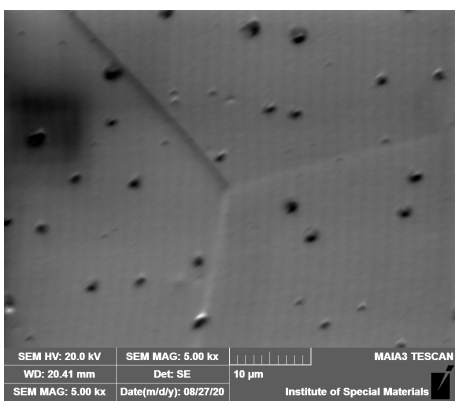

(a)

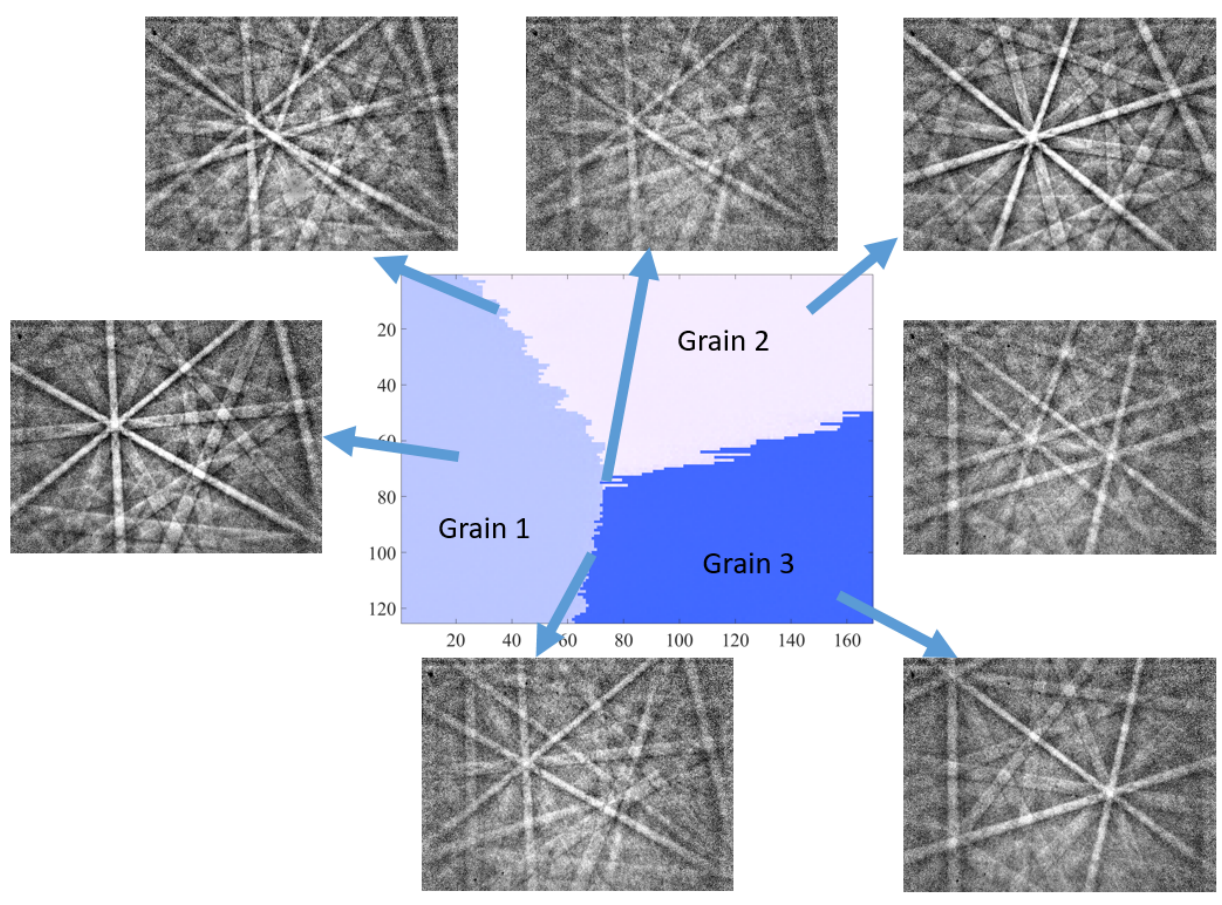

(b)

Figure 4: (a) SE image of the indexed triple grain area. (b) Inverse pole figure map of the triple grain boundary and corresponding EBSPs. Three net EBSPs and their mutually overlapped EBSPs are shown.

The EBSPs of the triple grains are indexed by both IDIC EBSD and IDIC-O EBSD algorithms. Figure 5(a)+5(c) correspond to PC fields when initializing the indexation by only one triplet of Euler angles. PC field variations are very limited, as the EBSD acquisition area is small. Note that $z^{*}$ values are higher for the upper region, contrary to that reported in Ref. [17. This is due to the contamination of the sample surface during the EBSD acquisition. 
EBSPs of the lower region, exposed to more contamination, tend to be more 'fuzzy' than the EBSPs of upper region. As a result, the $z^{*}$ value for blurry EBSPs decreases and surpasses the real $z^{*}$ trend related to the geometry. Figure $5(\mathrm{~d})$ shows the residual map, and Figure $5(\mathrm{e})$ the KAM field of IDIC EBSD calibration.

The Kernel Average Misorientation (KAM), or local misorientation, is a property that characterizes the local deformation level of a crystal [37. In the present case, the KAM is defined as the mean misorientation with its 4 closest neighbors. Normally in the calculatikon of KAM a cutoff threshold, say $5^{\circ}$ or $10^{\circ}$, is necessary to eliminate the effect of grain boundaries. However with IDIC-O EBSD this cutoff is not needed, as we can choose the corresponding Euler angle triplet in KAM calculation since both crystal orientations across grain boundaries are now available. As the sample is unstrained and the EBSD step size is small $(51 \mathrm{~nm})$, the true KAM value is assumed to be 0 , thus the measured KAM field indicates the uncertainty of crystal orientation indexation by IDIC for both grain interiors and grain boundaries. 


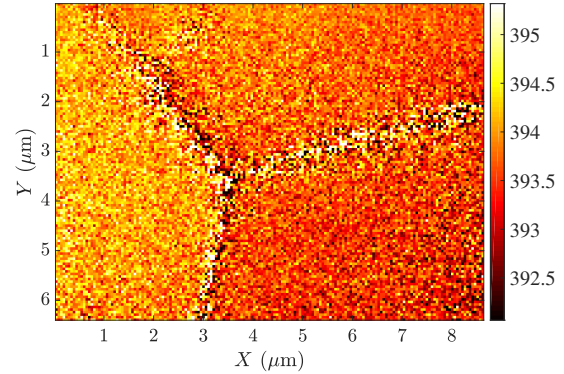

(a) $x^{*}$

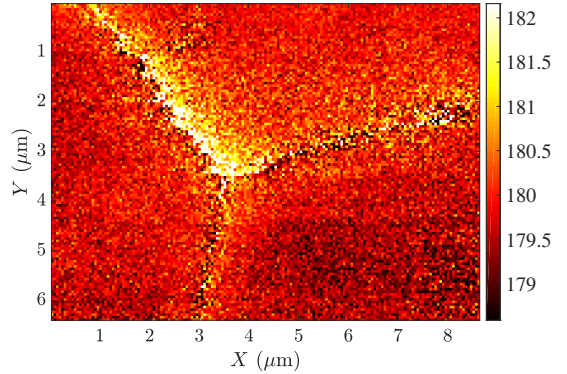

(b) $y^{*}$

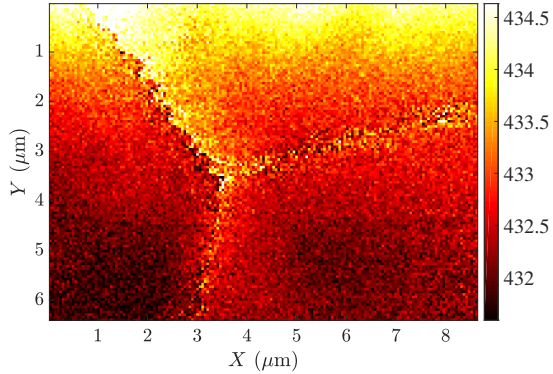

(c) $z^{*}$

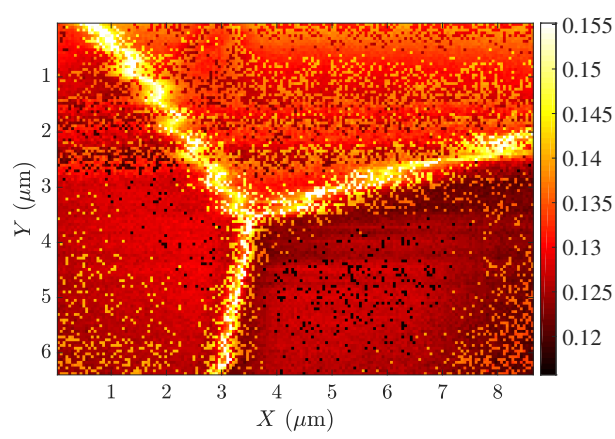

(d) Residual

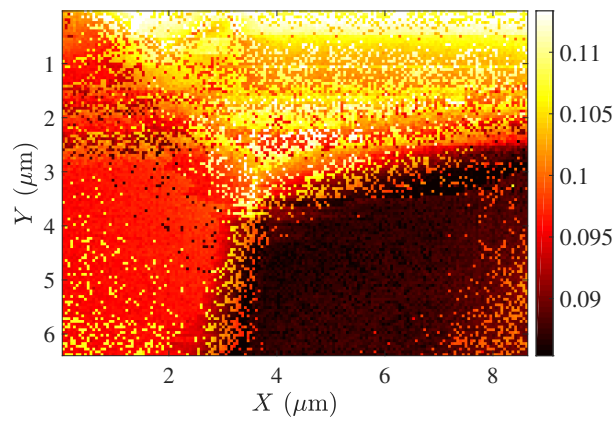

(f) IDIC-O Residual

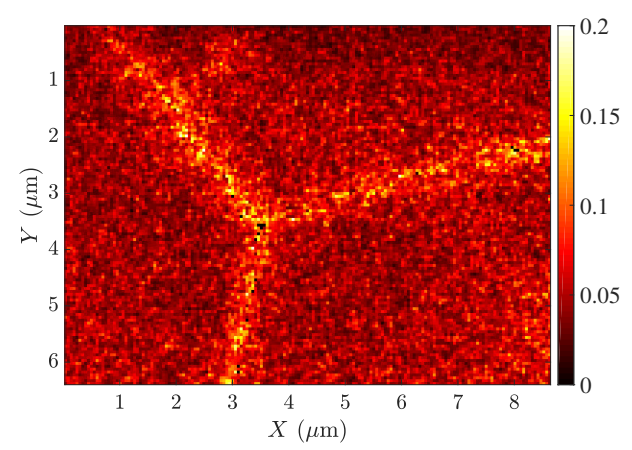

(e) KAM

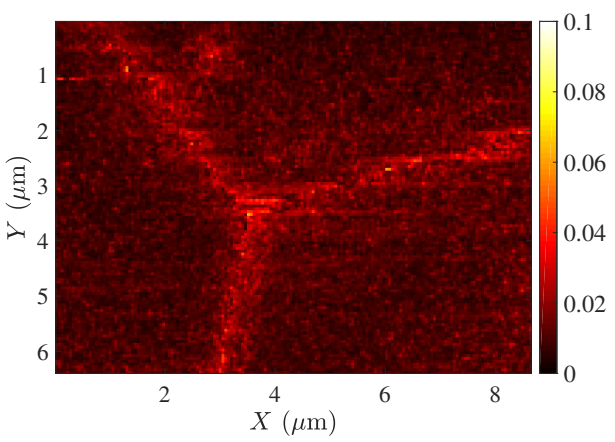

(g) IDIC-O KAM

Figure 5: Comparison of IDIC EBSD and IDIC-O EBSD for a fine EBSD acquisition at triple-joint grain boundary. Projection center components $x^{*}(\mathrm{a}), y^{*}(\mathrm{~b}), z^{*}(\mathrm{c})$, residual map (d) and KAM (e) calculated from IDIC EBSD with only one triplet of Euler angles. See Figure 1 for the definition of $x^{*}, y^{*}$ and $z^{*}$. Residual map (f) and KAM map (g) calculated from IDIC-O EBSD with up to three triplets of Euler angles.

Each component of $\mathrm{PC}$ as a function of $(X, Y)$ is linearly fitted and then set to its fitted value, before a secondround indexation is performed without updating PC values. Figure $5(\mathrm{f})-5(\mathrm{~g})$ show the resulting residual and KAM maps of IDIC-O EBSD. The uncertainty in crystal orientation, estimated as the median value of KAM for the unstrained sample, in grain interiors has fallen to $0.011^{\circ}$, i.e., a much lower value than the higher-resolution one reported in Ref. [17. Knowing that the same sample is used for the two EBSD acquisitions, this drop of KAM is mainly due to the reduced step size [38] and the master pattern generated from experimental EBSPs. When only one triplet of Euler angles is adopted, the residual values at grain boundaries are much higher than the intragrain points, as shown in Figure 5(d). However, when multiple triplets of Euler angles are used in IDIC-O EBSD 
calibration, the residuals at the GBs are no longer higher than intra-grain points as shown in Figure $5(\mathrm{f})$, implying that the exploitation of EBSP information achieves a comparable level for intra-grain pixels and near-boundary pixels. This comparison shows that considering the multiple Euler angles in overlapped EBSPs prevents inaccurate indexation results.

Figure 6 shows the contribution factor field of each crystal orientation. It is found that the contribution percentage varies gradually from 1 to 0 when a grain boundary is crossed, and the transition is quite smooth. This phenomenon is very close to that reported in Ref. [21, yet the sum of contributions is fixed as 1, which is more physical. Wavy forms along $X$ can be found in Figures 5(d) and 6(a) This phenomenon is not physical, as the grain boundaries are generally straight to minimize surface energy, which is also verified by the SE image of Figure 4(a), This wavy field is probably due to the scanning error of the EBSD rastering. Note that the scan error of numerous SEM modalities, including EBSD, is the most prominent in repositioning the electron beam after the acquisition of one line has been completed, or the drift in slow-scan direction. The wavy structure in Figure 6 is caused by this slow-scan drift. Scan error of EBSD has been studied and corrected by image correlation with other types of SEM images [39, 40, 41]. Due to the high sensitivity of contribution factor, IDIC-O EBSD helps to reveal the scan error without recourse to SE/BSE images. In fact, this wavy fluctuation should also appear in the $x^{*}$ field of Figure 5(a), yet this variation around $0.2 \mu \mathrm{m}$ (or 0.005 EBSP detector pixel) is beyond the sensitivity level of the PC calibration.

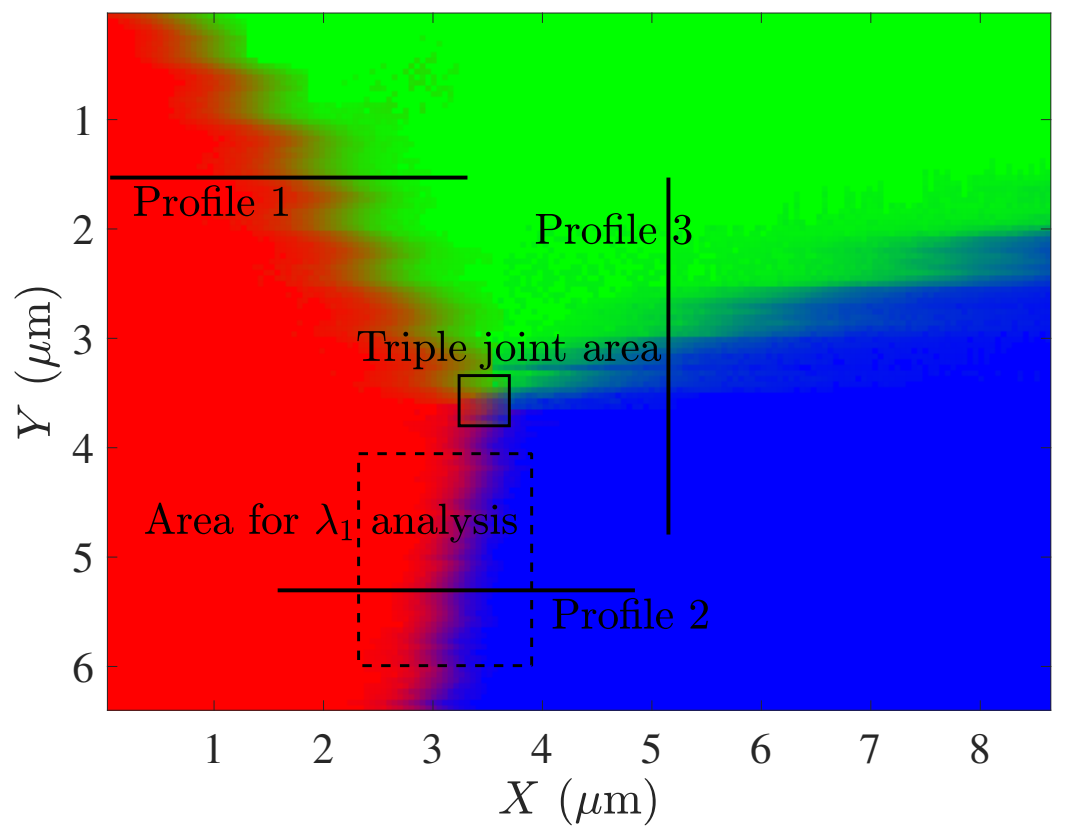

(a)

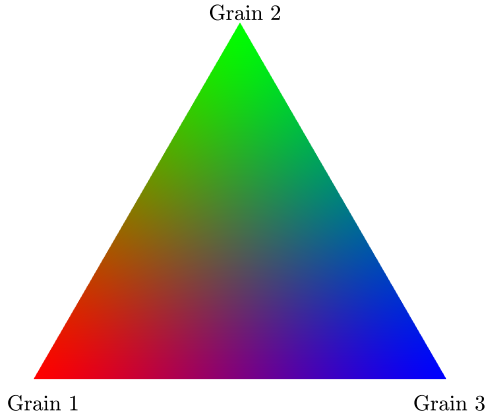

(b)

Figure 6: (a) The map of contribution ratio to each EBSP with several regions and profiles highlighted for further analyses. The colormap of $\lambda$ distribution is shown in (b).

Near the triple joint (marked in Figure 6(a), IDIC-O EBSD is performed with 3 triplets of Euler angles, and Figure 7 shows the contributing factors of each crystal orientation. Even with 14 degrees of freedom, IDIC-O EBSD converges well and captures the gradual transition between the three crystal orientations. 


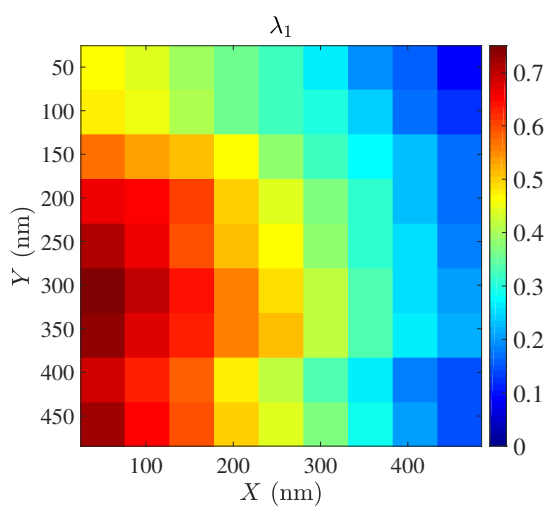

(a)

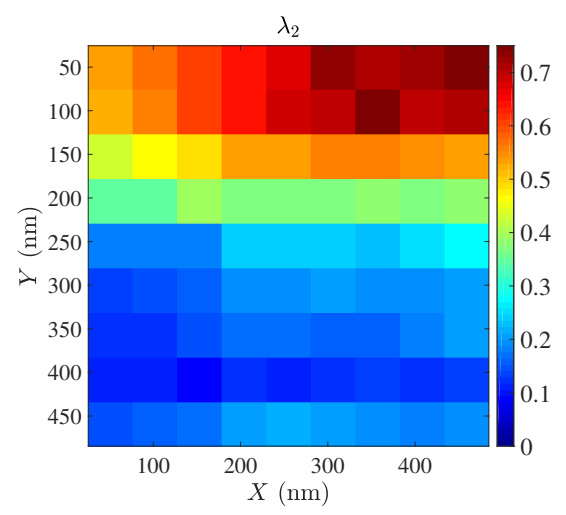

(b)

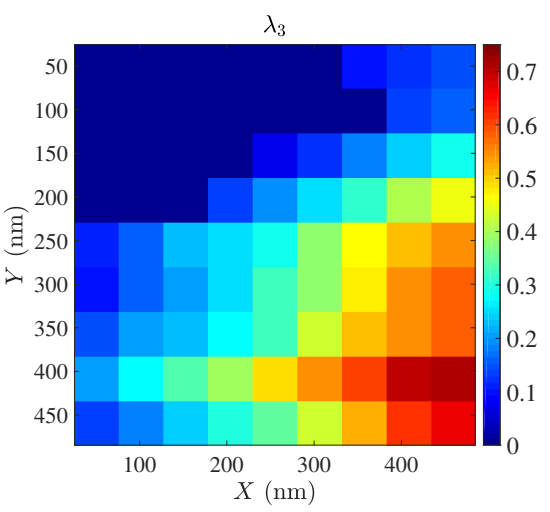

(c)

Figure 7: The contributing ratio of three grains to the overlapped EBSPs at the triple point.

Figure 8 shows the spatial density in natural logarithmic scale of emitting backscattering electrons (BSE) of the $70^{\circ}$-tilted $\mathrm{Al}-\mathrm{Mg}$ sample simulated by CASINO, a Monte-Carlo based open-source software for electron-solid interaction [42]. The accelerating voltage of the incident beam is $20 \mathrm{kV}$, and BSEs of kinematic energy lower than 18 $\mathrm{keV}$ are not counted. Only the emitting point is presented in Figure 8 (trajectories not shown). The BSE-emitting area is roughly a slim circular sector of height (radius) $0.7 \mu \mathrm{m}$ and base width $0.35 \mu \mathrm{m}$, which is comparable with the width of the $\lambda$ transition shown later in this section. This simulation agrees with the varying $\lambda$ transition area width described below, and hence, validates the method.

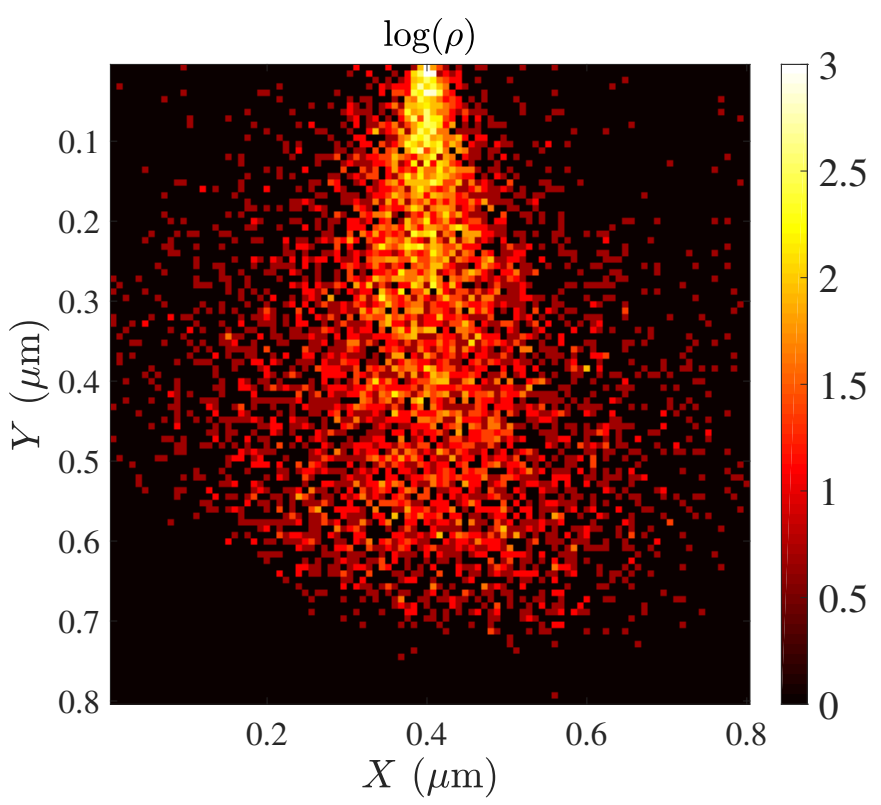

Figure 8: Density in logarithmic scale of emitting backscattered electrons on $70^{\circ}$ tilted $\mathrm{Al}-\mathrm{Mg}$ alloy sample, simulated with $20 \mathrm{kV}$ accelerating voltage by CASINO v2.5.1

Interestingly, the width of the $\lambda$ transition at the grain boundaries is $2.5,1.4$ and $1.0 \mu \mathrm{m}$ for the three grain boundary segments. Figure 9 shows the three profiles of misorientation and contribution factor across the three marked grain boundaries in Figure 6(a), As the contribution ratio of a crystal orientation drops in the overlapped 
EBSP, the indexed Euler angle drift increases for this crystal orientation. In terms of orders of magnitude, the orientation drift is around $0.3^{\circ}$ if the orientation contributes $20 \%$ to the overlapped EBSP. However, for very low contribution ratios $(\lambda<0.1)$, the accuracy of the corresponding crystal orientation could be very limited. For example, the crystal orientation drift of grain 1 in profile 1 increases to around $2^{\circ}$ when $\lambda_{1}<0.1$, which is beyond the y-axis limit of Figure 9(a) Interestingly, at the grain boundary, where two crystal orientations contribute equally to the overlapped EBSP, both the indexed orientations have a misorientation below $0.2^{\circ}$ with the grain interiors. Note that this misorientation level is lower than that reported in Ref. 20]. The long-distance orientation drift from grain interior to grain boundary could either be physical (real), or artificial when caused by the gray level modifications due to the appearance of neighbouring grains in the interaction volume. As a result, this orientation drift is not chosen as a metric of crystal orientation indexation uncertainty, which is discussed in more details below. It is suggested to define the grain boundary as the line where two crystal orientations contribute equally to the overlapped EBSP, and on each side, the crystal orientation is defined as the dominant orientation in the EBSP.

The varying crystal orientation transition width at grain boundaries is an interesting phenomenon. By applying a threshold of $5 \%$ to the contribution factor $\lambda$ and omitting smaller values, the $\lambda$ transition width between 0 and 0.5 for the three profiles are respectively $1.3 \mu \mathrm{m}, 0.5 \mu \mathrm{m}$ and $0.7 \mu \mathrm{m}$. As shown by the BSE emission density in Figure 8 , horizontal grain boundaries should have larger transition width than vertical ones. This relation is well observed for the GB $1 / 3$ and 2/3. However, the transition width for GB $1 / 2$ is larger than the others, though the GB inclination is on the IPF map is between those of GB $1 / 3$ and 2/3. This contrast implies that the GB $1 / 2$ is more tilted under surface than GB $1 / 3$ and $2 / 3$. The profiles of contributing factor $\lambda$ across grain boundaries have the potential to characterize the sub-surface microstructures, for example the inclination angle of grain boundaries 43, which is beyond the scope of the current paper. Note that the $\lambda$ profiles are very smooth for GB $1 / 2$ and $1 / 3$, while $\lambda$ shows fluctuations in Figure 9(c) due to the EBSD scan errors. In fact, its fluctuation of every $0.5 \mu \mathrm{m}$ will be visualized more clearly later in this section. By the IDIC-O EBSD algorithm, the spatial resolution of EBSD could be more precisely evaluated. As stated by Zaefferer 44, EBSD spatial resolution could be split as physical resolution and effective resolution. Here the found $0.5 \mu \mathrm{m}$ and $0.7 \mu \mathrm{m}$ are upper limits of physical resolutions for parallel and normal to the tilt axis. 


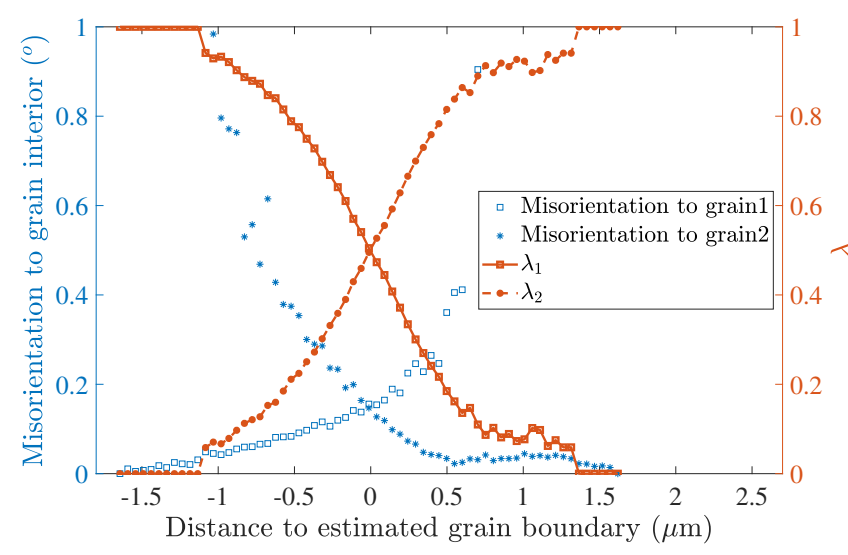

(a)

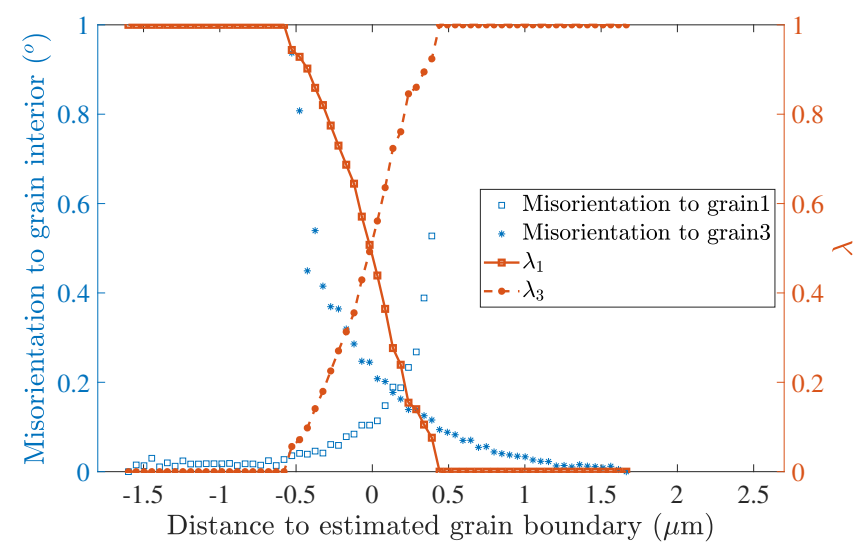

(b)

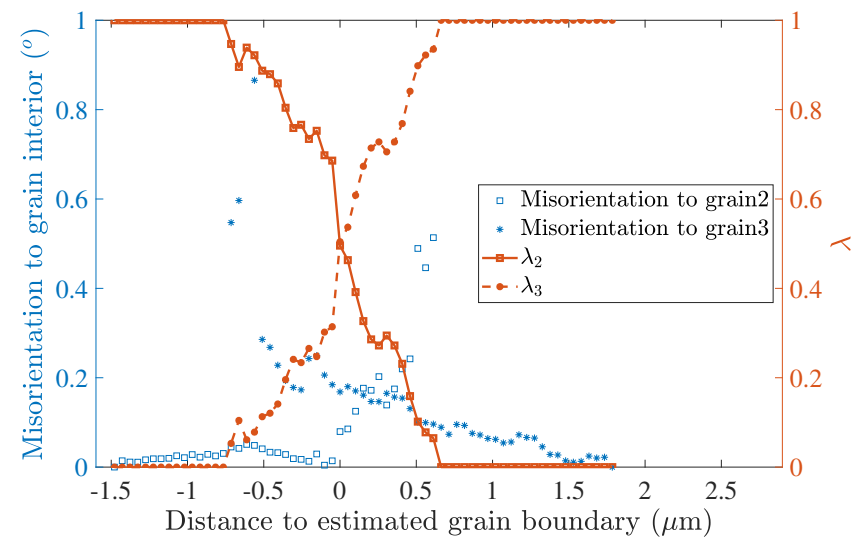

(c)

Figure 9: The misorientation and contribution factor retrieved by IDIC-O EBSD across the grain boundary of profile 1 (a), 2 (b) and 3 (c) shown in Figure 6.

Figures 10(a) and 10(b) show the enlarged KAM field near the grain boundary at the top left of Figure 6(a) for grains 1 and 2 respectively. Note that due to the overlapped EBSPs, the middle area is shared between both grains. Three profiles of $\lambda$ values $(0.25,0.5$ and 0.75$)$ are drawn in both figures. The profiles are very similar in shape and have a nearly constant horizontal shift, indicating a good precision of $\lambda$. Again, the wavy shape of $\lambda$ profiles are due to the scan error of EBSD. It is clear that as the contribution ratio of a crystal orientation drops in the overlapped EBSP, so does the indexed orientation precision. More precisely, the indexed orientation uncertainty is around $0.05^{\circ}$ if the orientation contributes $20 \%$ to the overlapped EBSP. Along the line where two crystal orientations contribute equally to the overlapped EBSP, the indexed orientation uncertainty is about $0.03^{\circ}$. For grain interiors, the uncertainty of indexation is estimated to be $0.011^{\circ}$ as shown in Figure $5(\mathrm{~g})$, For grain boundaries, the uncertainty level is the above mentioned $0.03^{\circ}$. This precision is quite satisfactory, knowing that the Hough-indexation, even by recognizing more Kikuchi bands than usual, has a precision around $0.4^{\circ}$ for equally mixed patterns [20]. 


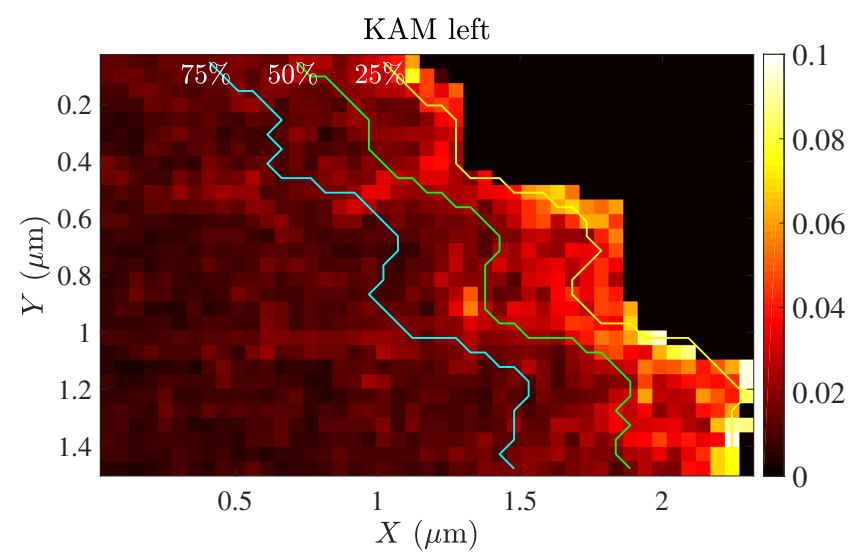

(a)

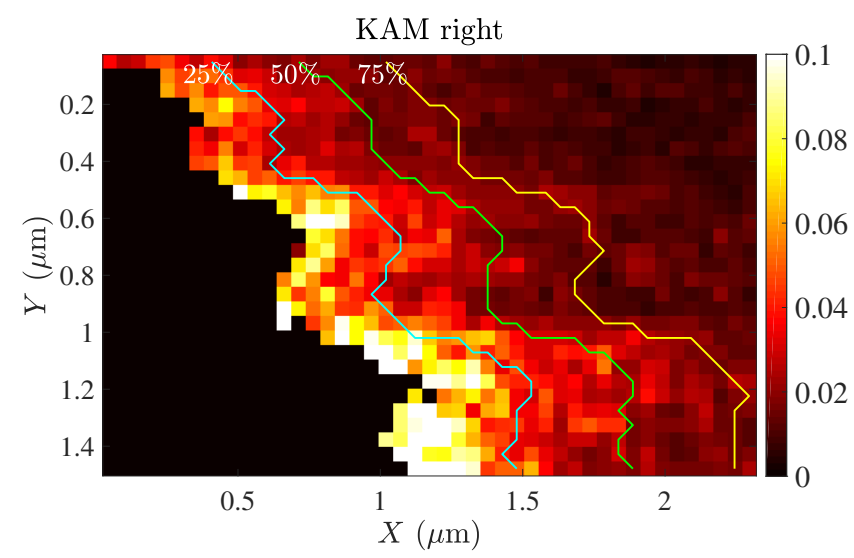

(b)

Figure 10: Kernel average misorientation retrieved by IDIC-O EBSD for grain 1 (a) and grain 2 (b). The profiles of contribution ratio $25 \%, 50 \%$ and $75 \%$ are drawn on both figures.

The contribution ratio of grain 1 at the boundary with grain 3 is analyzed to quantify the uncertainty of $\lambda_{1}$. A rectangular zone highlighted by dashed rectangle in Figure 6(a) is selected, where the the waviness of $\lambda$ is limited. $\lambda_{1}$ is fitted linearly, as shown in Figure 11(a). The residuals of $\lambda_{1}$ after the linear fitting is shown in Figure 11(b) Horizontal hot (hot) lines can be seen in Figure 11(b), which again are due to the scan error of EBSD. Note that the distance between those hot lines are roughly $0.5 \mu \mathrm{m}$, the same as that shown in Figure 9(c) and Figure 10. Figure 11(c) shows the histogram of $\Delta \lambda_{1}$ after deleting the aforementioned hot lines. For $91 \%$ EBSPs, the absolute value of $\Delta \lambda_{1} \operatorname{abs}\left(\Delta \lambda_{1}\right)$ is below 0.05 . Besides, the median value of $\operatorname{abs}\left(\Delta \lambda_{1}\right)$ is 0.022 . This demonstrates the good sensitivity of IDIC-O EBSD for indexing diffraction patterns at grain boundaries. As IDIC-O EBSD excels at distinguishing the contribution ratio of each crystal orientation, the effective resolution is significantly reduced. For example, as $\lambda_{1}$ varies near linearly between 0 and 0.5 at a distance of $0.5 \mu \mathrm{m}$ (see Figure 9(b)], $\lambda$ uncertainty of 0.022 means that the effective lateral resolution is $22 \mathrm{~nm}$ for $\mathrm{Al}$ at acceleration voltage of $20 \mathrm{kV}$. Note that this value is comparable to the resolution $30 \mathrm{~nm}$ for iron reported in Ref. [44], yet the fact that $\mathrm{Al}$ is a lighter element shows that IDIC-O EBSD improves the effective lateral resolution of EBSD. 


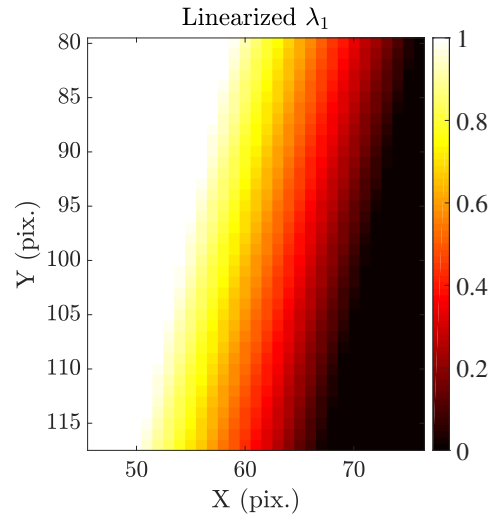

(a)

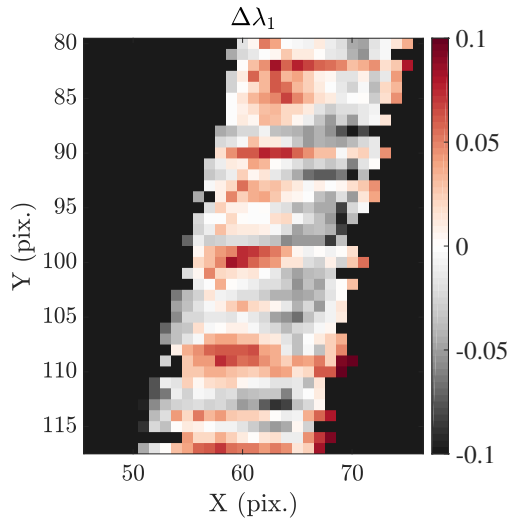

(b)

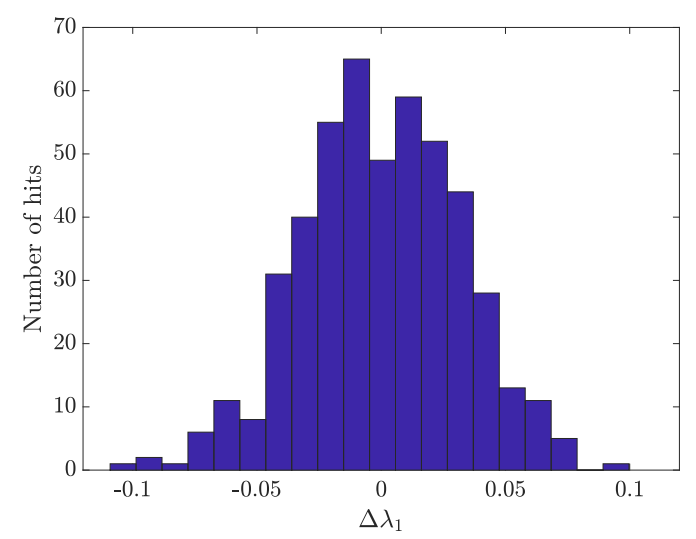

(c)

Figure 11: Uncertainty analysis of contribution factor $\lambda$. (a) Linear fitting of $\lambda_{1}$ at the boundary with grain 3 .

(b) Residual of $\lambda_{1}$ after linear fitting. (c) Histogram of $\lambda_{1}$ residuals after removing stray data.

Note that the sample is unstrained, and the EBSP resolution $600 \times 800$ pixels is higher than most fast EBSD acquisitions. The precision data reported here benchmarks the performance in an almost ideal case. As IDIC-O EBSD extracts more parameters from an EBSP than IDIC EBSD, it is more sensitive to Kikuchi band qualities. For highly-strained samples whose EBSPs show few Kikuchi bands, IDIC-O indexation results would not be credible.

\section{Test on an EBSD dataset on a strained polycrystal sample}

A more complex experiment has been performed to test the robustness of the proposed overlapped EBSP indexation method on a strained sample. An Aluminium-Magnesium (6\% wt.) sample was cold-rolled to a $10 \%$ reduction in thickness, followed by mechanical polishing and electropolishing in a 1:9 (volume fraction) solution of ethanol and perchloric acid. A high-definition EBSD acquisition was performed in Tescan MAIA3. The working distance for the EBSD acquisition was $16.3 \mathrm{~mm}$, electron acceleration voltage $20 \mathrm{kV}$, the probe current $20 \mathrm{nA}$, and the dwelling time 0.8 s. EBSD patterns were recorded with Bruker $\mathrm{e}^{-}$Flash $^{H D}$ detector at full definition (i.e., $1200 \times 1600$ pixels), and each pattern was the result of averaging three frames. A rectangular area of $1.03 \mathrm{~mm} \times 1.37 \mathrm{~mm}$ was indexed, and the step size was $6.87 \mu \mathrm{m}$, a large value as the grains are coarse.

Figure 12(a) shows the IPF map of this strained polycrystal Al-Mg sample, where the slip bands are readily visible. IDIC-O EBSD algorithm was applied to the EBSPs at grain boundaries. The step size being large, to capture the transition of EBSPs at grain boundaries, 10 EBSPs across a nearly vertical GB are chosen, as highlighted by the black line in Figure 12(a). The intersection of the profile and the grain boundary is noted as point P. Figure 12(b) shows the profiles of $\lambda$ and misorientation for the analyzed EBSPs. As opposed to the profiles of unstrained sample, the misorientation profiles are higher (in the order of $2-3^{\circ}$ ), even before the overlap of EBSPs. Besides, the transition of $\lambda$ remains smooth, indicating the successful application of IDIC-O EBSD on this strained sample when orientation gradients are present. 


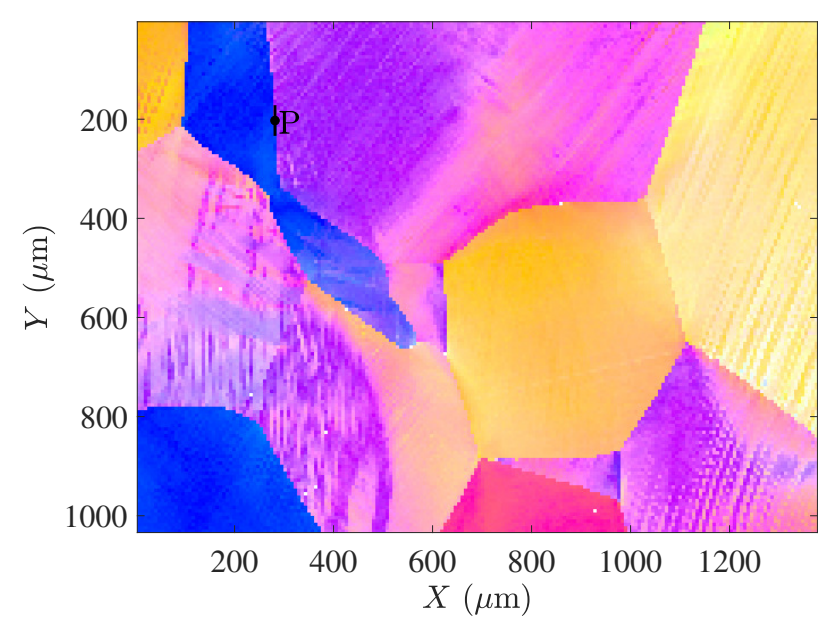

(a)

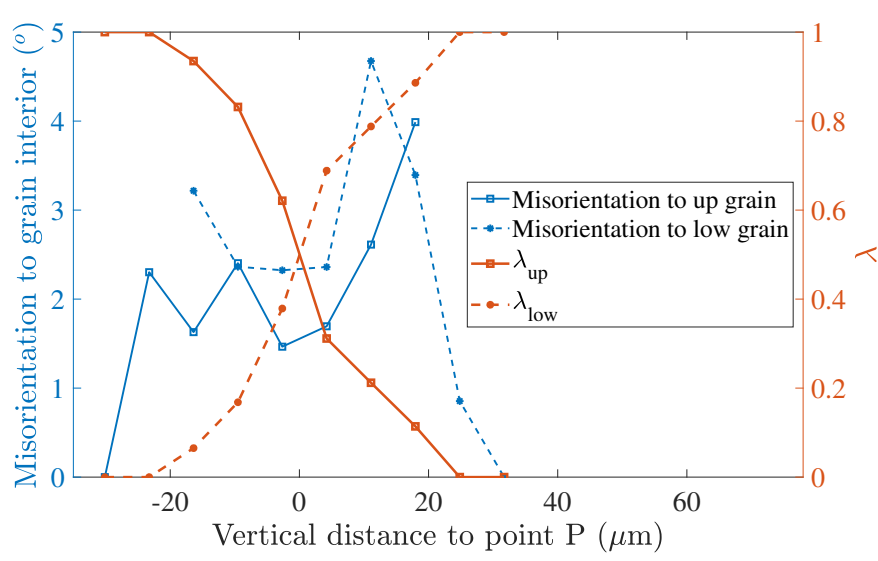

(b)

Figure 12: Results of the IDIC-O EBSD indexation on a strained sample. (a) The IPF map of the sample, with a black line highlighting the 10 EBSPs across a grain boundary. (b) The profiles of contribution ratios $\lambda$ and misorientation to grain interiors of the analysed EBSPs.

In theory, IDIC-O handles very well EBSD acquisitions with orientation gradients. In fact, this is exactly one of the key advantages of IDIC-O EBSD over cross-correlation based overlapped EBSP analysis introduced in Refs 20, 22]. As IDIC-O EBSD adopts a master pattern for registering experimental EBSPs, it can deal with any orientation at the grain boundaries, regardless of its misorientation with the grain interiors.

The proposed algorithm IDIC-O, which is adapted to overlapped EBSP indexation, could be extended to several other types of diffraction images (e.g., Kossel, Laue, transmission Kikuchi diffraction, or TEM diffraction). These techniques derive from the same principles of projection of diffracted beams and after a simple modification, IDIC-O EBSD could be applied to those overlapped images as well. Integrated algorithms for overlapped Laue and TEM diffraction, with all the above cited advantages, would bring significant gains to their exploitations. Due to the larger beam width of X-ray and larger interaction volume, the proposed algorithm could be employed more frequently in Laue diffraction.

\section{Conclusion}

This paper focused on indexing EBSPs with overlapped Kikuchi bands by full pattern matching. In doing so, a recently proposed integrated DIC EBSD indexing framework was extended to incorporate multiple crystal orientations. Through two high-definition experimental EBSD datasets, the performance of the algorithm has been tested.

The proposed algorithm has the following advantages:

1. Rich results. The directly retrieved results include multiple triplets of Euler angles, one triplet of pattern center coordinates and the contribution percentages of each crystal orientation. These results help to define the grain boundary with a much enhanced resolution, to characterize the spatial resolution of EBSD, to reveal possible scanning error in EBSD. For example, the radius of interaction volume, i.e., the physical lateral 
resolution, of EBSD acquisition at $20 \mathrm{kV}$ on $\mathrm{Al}-\mathrm{Mg}$ alloy has been evaluated to be $0.5 \mu \mathrm{m}$ in nearly vertical direction and increases to $0.7 \mu \mathrm{m}$ in nearly horizontal direction.

2. Low uncertainty. The strategy of global correlation reduces the measurement uncertainty. For example, in the test case with $600 \times 800$ pixels EBSPs on an annealed polycrystal $\mathrm{Al}$ alloy sample, the uncertainty on the contribution ratio is 0.02 and the uncertainty in crystal orientation indexation is below $0.03^{\circ}$ for overlapped EBSPs. Similar to IDIC EBSD calibration, the IDIC-O EBSD algorithm takes the entire EBSP in the optimization, and is thus optimal in terms of being less sensitive to white Gaussian noise corrupting diffraction images. Scan errors around $0.1 \mu \mathrm{m}$ can easily be recognized in the $\lambda$ field. The algorithm's performance remains untainted when orientation gradients exit.

3. The residual field of the correlation technique is intrinsically obtained by integrated DIC, which is not naturally calculated using a Hough transformation or dictionary indexing approaches. The residual field is a good indicator of the indexation quality. The information contained in the residual maps can be exploited in different ways, such as indicating which Kikuchi band system is not fully simulated.

Overlapped EBSPs have long been regarded as degraded diffraction patterns that reduces the orientation indexation precision at grain boundaries, where the information would be very valuable. Yet the present paper demonstrates that overlapped EBSPs are valuable sources of information. When processed properly, they provide twice as much information with a higher precision. This method opens the possibility to study more precisely various phenomena at grain boundaries using EBSD.

\section{Acknowledgements}

This work is financially supported by the Natural Science Foundation of China [Nos. 51901132, 51701120]. We thank Dr. Yang in Bruker China for her assistance in EBSD related Bruker software usages. We are also grateful to the financing of TESCAN CHINA, Ltd.

\section{Data availability}

The raw/processed data required to reproduce these findings cannot be shared at this time as the data also forms part of an ongoing study. 


\section{References}

[1] M. Kamaya. Influence of bulk damage on crack initiation in low-cycle fatigue of 316 stainless steel. Fatigue $\mathcal{G}^{3}$ Fracture of Engineering Materials \& Structures, 33(2):94-104, 2010.

[2] K. D. Ralston and N. Birbilis. Effect of grain size on corrosion: A review. CORROSION, 66(7):075005-075005$13,2010$.

[3] W.Z. Abuzaid, M.D. Sangid, J.D. Carroll, H. Sehitoglu, and J. Lambros. Slip transfer and plastic strain accumulation across grain boundaries in Hastelloy X. Journal of the Mechanics and Physics of Solids, 60(6):1201-1220, 2012 .

[4] S.I. Wright and B.L. Adams. Automatic analysis of electron backscatter diffraction patterns. Metallurgical Transactions A, 23(3):759-767, 1992.

[5] Y.H. Chen, S.U. Park, D. Wei, G. Newstadt, M.A. Jackson, J.P. Simmons, M. De Graef, and A.O. Hero. A dictionary approach to electron backscatter diffraction indexing. Microscopy and Microanalysis, 21(3):739-752, 2015.

[6] F. Ram, S. Wright, S. Singh, and M. De Graef. Error analysis of the crystal orientations obtained by the dictionary approach to EBSD indexing. Ultramicroscopy, 181(Supplement C):17 - 26, 2017.

[7] M. Jackson, E. Pascal, and M. Graef. Dictionary indexing of electron back-scatter diffraction patterns: a hands-on tutorial. Integrating Materials and Manufacturing Innovation, 8, 052019.

[8] W.C. Lenthe, S. Singh, and M. De Graef. A spherical harmonic transform approach to the indexing of electron back-scattered diffraction patterns. Ultramicroscopy, 207:112841, 2019.

[9] D. Jha, S. Singh, R. Al-Bahrani, W. Liao, A. Choudhary, M. De Graef, and A. Agrawal. Extracting grain orientations from EBSD patterns of polycrystalline materials using convolutional neural networks. Microscopy and Microanalysis, 24(5):497-502, 2018.

[10] Y. Shen, R. Pokharel, T.J. Nizolek, A. Kumar, and T. Lookman. Convolutional neural network-based method for real-time orientation indexing of measured electron backscatter diffraction patterns. Acta Materialia, 170:118 - 131, 2019.

[11] Z. Ding, E. Pascal, and M. De Graef. Indexing of electron back-scatter diffraction patterns using a convolutional neural network. Acta Materialia, 199:370 - 382, 2020.

[12] F. Ram and M. De Graef. Phase differentiation by electron backscatter diffraction using the dictionary indexing approach. Acta Materialia, 144:352 - 364, 2018.

[13] S. Singh, F. Ram, and M. De Graef. Emsoft: Open source software for electron diffraction/image simulations. Microscopy and MicroAnalysis, 23:S1:212-213, 2017. 
[14] G. Nolze, M. Jürgens, J. Olbricht, and A. Winkelmann. Improving the precision of orientation measurements from technical materials via EBSD pattern matching. Acta Materialia, 159:408 - 415, 2018.

[15] E.L. Pang, P.M. Larsen, and C.A. Schuh. Global optimization for accurate determination of EBSD pattern centers. Ultramicroscopy, 209:112876, 2020.

[16] T. Tanaka and A.J. Wilkinson. Pattern matching analysis of electron backscatter diffraction patterns for pattern centre, crystal orientation and absolute elastic strain determination - accuracy and precision assessment. Ultramicroscopy, 202:87 - 99, 2019.

[17] Q. Shi, D. Loisnard, C. Dan, F. Zhang, H. Zhong, H. Li, Y. Li, Z. Chen, H. Wang, and S. Roux. Calibration of crystal orientation and pattern center of EBSD using integrated digital image correlation. Materials Characterization, 178:111206, 2021.

[18] T. Friedrich, A. Bochmann, J. Dinger, and S. Teichert. Application of the pattern matching approach for EBSD calibration and orientation mapping, utilising dynamical EBSP simulations. Ultramicroscopy, 184:44 $51,2018$.

[19] A. Winkelmann, B.M. Jablon, V.S. Tong, C. Trager-Cowan, and K.P. Mingard. Improving EBSD precision by orientation refinement with full pattern matching. Journal of Microscopy, 277(2):79-92, 2020.

[20] S.I. Wright, M.M. Nowell, R. De Kloe, and L. Chan. Orientation precision of electron backscatter diffraction measurements near grain boundaries. Microscopy and Microanalysis, 20(3):852-863, 2014.

[21] J. Kacher, B. Adams, D. Fullwood, and C. Landon. Separating Coincident Electron Backscatter Diffraction Patterns Near Interfaces, chapter 15, pages 147-154. John Wiley \& Sons, Ltd, New Jersey, 2008.

[22] V. Tong, J. Jiang, A. J. Wilkinson, and T. B. Britton. The effect of pattern overlap on the accuracy of high resolution electron backscatter diffraction measurements. Ultramicroscopy, 155(Supplement C):62 - 73, 2015.

[23] S. Singh and M. De Graef. Dictionary indexing of electron channeling patterns. Microscopy and Microanalysis, 23(1):1-10, 2017.

[24] W. Lenthe, L. Germain, M. Chini, N. Gey, and M. Graef. Spherical indexing of overlap ebsd patterns for orientation-related phases - application to titanium. Acta Materialia, 188, 022020.

[25] F. Hild and S. Roux. Digital image correlation: from displacement measurement to identification of elastic properties - a review. Strain, 42(2):69-80, 2006.

[26] S. Roux and F. Hild. Stress intensity factor measurements from digital image correlation: post-processing and integrated approaches. International Journal of Fracture, 140(1):141-157, 2006.

[27] H. Leclerc, J.-N. Périé, S. Roux, and F. Hild. Integrated digital image correlation for the identification of mechanical properties. MIRAGE 2009, LNCS, 5496:161-171, 2009. 
[28] T. Vermeij and J.P.M. Hoefnagels. A consistent full-field integrated DIC framework for HR-EBSD. Ultramicroscopy, 191:44 - 50, 2018.

[29] T.J. Ruggles, G.F. Bomarito, R.L. Qiu, and J.D. Hochhalter. New levels of high angular resolution EBSD performance via inverse compositional Gauss-Newton based digital image correlation. Ultramicroscopy, 195:85 $-92,2018$.

[30] Q. Shi, S. Roux, F. Latourte, and F. Hild. Estimation of elastic strain by integrated image correlation on electron diffraction patterns. Ultramicroscopy, 199:16-33, 2019.

[31] C. Ernould, B. Beausir, J.-J. Fundenberger, V. Taupin, and E Bouzy. Integrated correction of optical distortions for global HR-EBSD techniques. Ultramicroscopy, page 113158, 2020.

[32] F. Ram and M. Graef. Energy dependence of the spatial distribution of inelastically scattered electrons in backscatter electron diffraction. Physical review. B, Condensed matter, 97:134104, 2018.

[33] A.P. Day. Spherical EBSD. Journal of Microscopy, 230(3):472-486, 2008.

[34] C. Zhu, K. Kaufmann, and K. Vecchio. Automated reconstruction of spherical kikuchi maps. Microscopy and Microanalysis, 25(4):912-923, 2019.

[35] H. Zhong, Q. Shi, Z. Chen, C. Dan, S. Zhong, and H. Wang. Residual-based pattern center calibration in high-resolution electron backscatter diffraction. Micron, 146:103081, 2021.

[36] F. Hild and S. Roux. Digital image correlation. In P. Rastogi and E. Hack, editors, Optical Methods for Solid Mechanics. A Full-Field Approach, pages 183-228. Wiley-VCH, Weinheim (Germany), 2012.

[37] A. J. Wilkinson and T. B. Britton. Strains, planes, and EBSD in materials science. Materials Today, 15:366$376,2012$.

[38] S.I. Wright, D.P. Field, and D.J. Dingley. Advanced Software Capabilities for Automated EBSD, pages 141-152. Springer US, 2000.

[39] Y. Zhang, A.B. Elbrønd, and F. Lin. A method to correct coordinate distortion in EBSD maps. Materials Characterization, 96:158-165, 2014.

[40] Q. Shi, S. Roux, F. Latourte, F. Hild, D. Loisnard, and N. Brynaert. On the use of SEM correlative tools for in situ mechanical tests. Ultramicroscopy, 184(Part A):71 - 87, 2018.

[41] V.S. Tong and T.B. Britton. TrueEBSD: Correcting spatial distortions in electron backscatter diffraction maps. Ultramicroscopy, 221:113130, 2021.

[42] D. Drouin, A.R. Couture, D. Joly, X. Tastet, V. Aimez, and R. Gauvin. CASINO v2. 42-a fast and easy-to-use modeling tool for scanning electron microscopy and microanalysis users. Scanning, 29(3):92-101, 2007.

[43] C. Sorensen, J. Basinger, M. Nowell, and D. Fullwood. Five-parameter grain boundary inclination recovery with ebsd and interaction volume models. Metallurgical and Materials Transactions A, 45, 082014. 
[44] S. Zaefferer. On the formation mechanisms, spatial resolution and intensity of backscatter Kikuchi patterns. Ultramicroscopy, 107(2-3):254 - 266, 2007. 